Dublin Ireland, 842 children were discharged with no fixed address, typically into emergency accommodation in 2018. This compares with 651 children in 2017, a 29\% increase. ${ }^{1-}$ Children born into homelessness are more likely to have low birth weights and are at greater risk of death. ${ }^{2}$ The aim of this study was to compare a random cohort of children presenting to the ED and compare differences between children living in homelessness and those at risk of homelessness to those living in stable accommodation across a variety of parameters.

Methods A self-administered parental questionnaire was handed to parents checking in to the ED in TSCUH over a 2 -week period. An information sheet was also given to parents to explain the purpose of the research. Results were analysed using Microsoft excel.

Results $120(n=120)$ questionnaires were filled out by a random selection of parents over the initial study period. Age range was 1 month to 15 years. Number of General Practice (GP) attendances ranged between 0 and 6 in past 6 months, ED ranged between 0 and 12 .

$50 \%(n=60)$ owned their own homes. 96\% $(n=115)$ were fully vaccinated. $89 \% \quad(n=106)$ thought their child had a nutritionally complete diet. However 24\% $(n=29)$ thought their living situation did not enable the parent to adequately prepare/cook meals for their child.

$18 \%(n=22)$ lived in homelessness/emergency accommodation or with family. In this group $27 \%(n=6)$ vs $19 \%(n=17)$ had fast-food/ready-meals twice or more per week. Parents thought their accommodation had a negative effect on their child's health in $19 \%(n=4)$ vs $6 \%(n=6)$ in this group. Making and maintaining friends was thought to be affected by accommodation in $20 \%(n=4)$ vs $7 \% \quad(n=7)$. The effect on ability to exercise/play of living situation was $36 \%(n=8)$ vs $12 \%(n=12)$.

Conclusion The above data clearly demonstrates parental perspectives on the impact of homelessness on children. This compares the perspectives of parents living in different types of accommodation and demonstrates the detrimental effects homelessness can have on children's health and well-being.

\section{P265 THE RELIABILITY OF POINT OF CARE KETONE MEASUREMENT IN THE PAEDIATRIC HYPOGLYCAEMIA SETTING}

John Coveney*, Sinead McGlacken Byrne, Nuala Murphy. Children's University Hospital, Temple St., Dublin, Ireland

\subsection{6/archdischild-2019-epa.615}

Introduction Point of care capillary ketone (beta-hydroxybutyrate) measurement is often used in the management of paediatric dysglycaemia. The accuracy of point of care ketone (POCK) measurement has not been evaluated in the setting of paediatric hypoglycaemia. This study aimed to assess the accuracy of POCK measurement compared to gold-standard laboratory plasma ketone (PK) measurement in a population of infants and children presenting to a paediatric emergency department.

Methods This retrospective study was conducted between January-December 2017 in the Emergency Department (ED) at Children's University Hospital (CUH), Temple Street, Dublin, Ireland. The ED recorded 54,222 attendances during the study period and all hypoglycaemia screens performed in the study period were reviewed.

PK values were extracted from laboratory records. POCK results were retrieved from patient's electronic emergency department medical records. Where data was incomplete (either POCK or PK measurement were not recorded), results were excluded from the analysis. Results were also excluded where greater than thirty minutes had elapsed between POCK and PK measurement. The agreement between the two methods of ketone measurement was analysed using a Bland-Altman plot. The difference between mean POCK and PK were analysed using a paired t-test.

Results A total of 34 hypoglycaemia screens were performed in ED during the study period. Of these, 15 screens were excluded (two because of incomplete data and 13 because of a time interval of greater than thirty minutes between POCK and PK measurement). 19 screens were included in the analysis. No significant difference was seen between mean PK $(3.39 \pm 1.66 \mathrm{mmol} / \mathrm{L})$ and mean POCK $(4.28 \pm$ $1.62)$, with a mean difference of $-0.30 \pm 0.54 \mathrm{mmol} / \mathrm{L}$ (95\% CI, -0.364 to $-0.243 ; \mathrm{p}=0.032$ ) No significant proportional bias was seen between POCK and PK levels on Band-Altman plot.

Discussion This study provides evidence for the use of POCK as a reliable surrogate for PK in the setting of hypoglycaemia in paediatric patients. Accurate POCK measurement is very useful in the diagnosis and management of paediatric hypoglycaemia. In the paediatric ED, children presenting with hypoglycaemia often do so during episodes of intercurrent illness where dehydration is common, and phlebotomy is challenging. Furthermore, when faced with a small child with hypoglycaemia, timely correction of hypoglycaemia is an urgent priority. While not a substitute for formal diagnostic tests, POCK measurements are quick and easy to obtain, can direct appropriate investigation and allow sample prioritisation where sample volume is limited.

\section{P266 B-LINES SCORE ON LUNGULTRASOUND AS A DIRECT MEASURE OF RESPIRATORY DYSFUNCTION IN PICU PATIENTS WITH SEVERE POLYTRAUMA}

Kateryna Dmytriieva, Oleksandr Katilov*, Dmytro Dmytriiev, Kostyantin Dmytriiev. Vinnitsa National Medical University, Vinnitsa, Ukraine

\subsection{6/archdischild-2019-epa.616}

Purpose Fluid overload is frequently found in critically ill pediatric patients with severe polytrauma (SP) and is associated with adverse outcomes. Lungultrasonography (LU) and bioimpedance spectroscopy (BIS) are potentially useful tools for the noninvasive volume assessment. We evaluated the utility of these measures, alone or in combination, in estimating the $\mathrm{PaO}_{2} / \mathrm{FiO}_{2}$ ratio in critical pediatric patients with SP .

Methods In a prospective pilot observational study we included 23 patients who presented on admission or developed at any time during intensivecare unit stay SP. Patients were studied at baseline and after $48 \mathrm{~h}$ with LU, BIS and arterial blood gas.

Results In the univariable analysis, the $\mathrm{PaO}_{2} / \mathrm{FiO}_{2}$ ratio was negatively correlated with the $\mathrm{B}$-lines score, and this 